\title{
Morphological diversity in tenrecs (Afrosoricida, Tenrecidae): comparing tenrec skull diversity to their closest relatives
}

Sive Finlay, Natalie Cooper

Morphological diversity is often studied qualitatively. However, to truly understand the evolution of exceptional diversity, it is important to take a quantitative approach instead of relying on subjective, qualitative assessments. Here, we present a quantitative analysis of morphological diversity in a Family of small mammals, the tenrecs (Afrosoricida, Tenrecidae).

Tenrecs are often cited as an example of an exceptionally morphologically diverse group. However, this assumption has not been tested quantitatively. We use geometric morphometric analyses of skull shape to test whether tenrecs are more morphologically diverse than their closest relatives, the golden moles (Afrosoricida, Chrysochloridae). Tenrecs occupy a wider range of ecological niches than golden moles so we predict that they will be more morphologically diverse.

Contrary to our expectations, We find that tenrec skulls are only more morphologically diverse than golden moles when measured in lateral view. Furthermore, similarities among the species-rich Microgale tenrec Genus appear to mask higher morphological diversity in the rest of the Family. These results reveal new insights into the morphological diversity of tenrecs and highlight the importance of using quantitative methods to test qualitative assumptions about patterns of morphological diversity. 


\section{PeerJ Reviewing Manuscript}

1 Sive Finlay ${ }^{1,2}$ and Natalie Cooper ${ }^{1,2, *}$

${ }_{2}^{1}$ School of Natural Sciences, Trinity College Dublin, Dublin 2, Ireland.

$3^{2}$ Trinity Centre for Biodiversity Research, Trinity College Dublin, Dublin

4 2, Ireland.

${ }_{5}$ *Corresponding author: Natalie Cooper; ncooper@tcd.ie; Zoology

6 Building, Trinity College Dublin, Dublin 2, Ireland. Tel: +353 1 8961926. 


\section{PeerJ Reviewing Manuscript}

\section{Introduction}

8 Analysing patterns of morphological diversity (the variation in physical form (Foote, 1997) has important implications for our understanding of ecological and evolutionary traits. Increasingly, many studies recognise the importance of quantifying the degree of morphological diversity instead of relying on subjective assessments of diversity in form (e.g. Ruta et al., 2013; Hopkins, 2013; Goswami et al. 2011; Drake and Klingenberg, 2010; Price et al., 2010; Brusatte et al., 2008). We need to quantify the morphological similarities and differences among species to gain a better understanding of their ecological interactions and evolutionary history.

Unfortunately, morphological diversity is difficult to quantify. Many studies are constrained to measuring the diversity of specific traits rather than overall morphologies (Roy and Foote, 1997). In addition, our perception of morphological diversity is influenced by the trait being measured, and results may depend on the particular trait being analysed (Foth et al., 2012). Furthermore, linear measurements of morphological traits can restrict our understanding of overall morphological variation; a distance matrix of measurements among specific points is unlikely to give a complete representation of a three dimensional structure (Rohlf and Marcus, 1993). Geometric morphometric approaches can circumvent some of these issues by using a system of Cartesian landmark coordinates to define anatomical points (Adams et al. 2004, and references therein). This method captures more of the true, overall 


\section{PeerJ Reviewing Manuscript}

anatomical shape of particular structures (Mitteroecker and Gunz, 2009). In particular, two-dimensional geometric morphometric approaches are commonly used to analyse $3 \mathrm{D}$ morphological shape and are appropriate for cross-species comparisons (e.g. Muschick et al., 2012; Panchetti et al. 2008; Wroe and Milne, 2007). Any bias from $2 \mathrm{D}$ representation of a ${ }_{3} \mathrm{D}$ structure is unlikely to be a significant issue for interspecific studies as the overall shape variation among species is geater than discrepancies introduced by using 2D morphometric techniques (Cardini, 2014). These more detailed approaches are useful tools for studying patterns of morphological diversity.

Here we apply geometric morphometric techniques to quantify morphological diversity in a family of small mammals, the tenrecs. Tenrecs (Afrosoricida, Tenrecidae) are a morphologically diverse group that researchers often identify as an example of both convergent evolution and an adaptive radiation (Soarimalala and Goodman, 2011; Eisenberg and Gould, 1969). The family is comprised of 34 species, 31 of which are endemic to Madagascar (Olson, 2013). Body masses of tenrecs span three orders of magnitude (2.5 to $>2,000 \mathrm{~g})$ : a greater range than all other families, and most orders, of living mammals (Olson and Goodman, 2003). Within this vast size range there are tenrecs which resemble shrews (Microgale tenrecs), moles (Oryzorictes tenrecs) and hedgehogs (Echinops and Setifer tenrecs, Eisenberg and Gould, 1969). The similarities among tenrecs and other small mammal species include examples of morphological, behavioural and ecological convergence (Soarimalala and Goodman, 2011). Tenrecs are one of only four endemic 


\section{PeerJ Reviewing Manuscript}

mammalian clades in Madagascar and the small mammal species they resemble are absent from the island (Garbutt, 1999). Therefore, it appears that tenrecs represent an adaptive radiation of species which filled otherwise vacant ecological niches through gradual morphological specialisations (Poux et al., 2008).

The claims that tenrecs are an example of both an adaptive radiation and convergent evolution have not been investigated quantitatively. There are qualitative similarities among the hind limb morphologies of tenrecs and several other unrelated species with similar locomotory styles (Salton and Sargis, 2009) but the degree of morphological similarity has not been established. Morphological diversity is an important feature of adaptive radiations (Losos and Mahler, 2010) and it also informs our understanding of convergent phenotypes (Muschick et al., 2012). Therefore, it is important to quantify patterns of morphological diversity in tenrecs to gain an insight into their evolution.

We present the first quantitative study of patterns of morphological diversity in tenrecs. We use geometric morphometric techniques (Rohlf and Marcus, 1993) to compare cranial morphological diversity in tenrecs to that of their closest relatives, the golden moles (Afrosoricida, Chrysochloridae). We expect tenrecs to be more morphologically diverse than golden moles because tenrecs occupy a wider variety of ecological niches. The tenrec family includes terrestrial, semi-fossorial, semi-aquatic and semi-arboreal species (Soarimalala and Goodman, 2011). In contrast, all golden moles occupy very similar, fossorial 
${ }_{85}$ Materials and Methods not as great as it first appears.

\section{Data collection}

ecological niches (Bronner, 1995). Greater ecological variety is often (though not always: McGee and Wainwright, 2013; Losos and Mahler. 2010) correlated with higher morphological diversity. However, our results reveal that, in skulls at least, morphological diversity in tenrecs is

Our methods involved i) data collection, ii) geometric morphometric analyses and iii) estimating morphological diversity. For clarity, Figure 1 summarises all of these steps and we describe them in detail below.

One of us (SF) collected data from five museums: Natural History

Museum, London (BMNH), Smithsonian Institute Natural History

Museum, Washington D.C. (SI), American Museum of Natural History,

New York (AMNH), Museum of Comparative Zoology, Cambridge M.A.

(MCZ) and Field Museum of Natural History, Chicago (FMNH). We used the taxonomy in Wilson \& Reeder's Mammal Species of the World (2005), except for the recently discovered tenrec species Microgale jobihely (Goodman et al., 2006). We photographed all of the intact tenrec and golden mole skulls available in the collections. This included 31 of the 34 species in the tenrec family (Olson, 2013) and 12 of the 21 species of golden moles (Wilson and Reeder, 2005). 


\section{PeerJ Reviewing Manuscript}

We took pictures of the skulls using photographic copy stands. To take possible light variability into account, we took a photograph of a white sheet of paper each day and used the custom white balance function on the camera to set the image as the baseline "white" measurement for those particular light conditions. We photographed the specimens with a Canon EOS 650D camera fitted with a EF $100 \mathrm{~mm}$ f/2.8 Macro USM lens and using a remote control (Hähnel Combi TF) to avoid camera shake. We photographed the specimens on a black material background with a light source in the top left-hand corner of the photograph and a scale bar placed below the specimen. We used small bean bags to hold the specimens in position to ensure that they lay in a flat plane relative to the camera, and used the grid-line function on the live-view display screen of the camera to position the specimens in the centre of each image.

All skulls were photographed in three views: dorsal, ventral and lateral (right side) (Figure 1). When the right sides of the skulls were damaged or incomplete, we photographed the left sides and later reflected the images (e.g. Barrow and Macleod, 2008). Some specimens were too damaged to use in particular views so there were a different total number of images for each analysis. Our final data sets included photographs of 182 skulls in dorsal view ( 148 tenrecs and 34 golden moles), 173 skulls in ventral view (141 tenrecs and 32 golden moles) and 171 skulls in lateral view (140 tenrecs and 31 golden moles). Details of the total sample size for each species can be found in the supplementary material. 


\section{PeerJ Reviewing Manuscript}

After taking the photographs we used the Canon Digital Photo Professional software (Canon, 2013) to convert the raw files to binary (grey scale) images and re-save them as TIFF files (uncompressed files preserve greater detail, RHOI, 2013). Photographs of the specimens from the American Museum of Natural History and the Smithsonian Institute Natural History Museum are available on figshare (dorsal; Finlay and Cooper (2013a), ventral; Finlay and Cooper $(2013 \mathrm{C})$ and lateral; Finlay and Cooper (2013b)). Copyright restrictions from the other museums prevent public sharing of their images but they are available from the authors on request.

\section{Geometric morphometric analyses}

We used a combination of landmark and semilandmark approaches to assess the shape variability in the skulls. We used the TPS software suite (Rohlf, 2013) to digitise landmarks and curves on the photos. We set the scale on each image individually to standardise for the different camera heights used when photographing the specimens. We created separate data files for each of the three morphometric analyses (dorsal, ventral and lateral views). One of us (SF) digitised landmarks and semilandmark points on every image individually.

Figure 2 depicts the landmarks and curves which we used for each skull view. For landmarks defined by dental structures, we used published dental sources where available (Repenning, 1967; Eisenberg and Gould, 1969; Nowak, 1983; MacPhee, 1987; Knox Jones and 
Manning, 1992: Davis and Schmidly, 1997: Quérouil et al. 2001;

Nagorsen, 2002; Wilson and Reeder, 2005; Goodman et al., 2006; Karataş

et al., 2007; Hoffmann and Lunde, 2008; Asher and Lehmann, 2008;

Muldoon et al., 2009: Lin and Motokawa, 2010) to identify the number and type of teeth in each species. Detailed descriptions of the landmarks, as well as an example figure of landmarks on golden mole skulls, can be found in the supplementary material.

When using semilandmark approaches there is a potential problem of over-sampling: simpler structures will require fewer semilandmarks to accurately represent their shape (MacLeod, 2012). To ensure that we applied a uniform standard of shape representation to each outline segment (i.e. that simple structures would not be over-represented and more complex features would not be under-represented), we followed the method outlined by MacLeod (2012) to determine the minimum number of semilandmark points which would give accurate representations of morphological shape. We used 54 points for skulls in dorsal view (10 landmarks, 44 semilandmarks across 4 curves), 73 points for skulls in ventral view (13 landmarks, 6o semilandmarks) and 44 points for skulls in lateral view (9 landmarks and 35 semilandmarks across 2 curves). See Figure 2 and the supplementary material for more details.

After creating the files with the landmarks and semilandmarks placed on each photograph, we used TPSUtil (Rohlf, 2012) to create "sliders" files that defined which points in the TPS files should be 
treated as semilandmarks (Zelditch et al., 2012). We combined the landmarks and taxonomic identification files into a single morphometrics data object and carried out all further analyses in $\mathrm{R}$ version 3.1.1 (R Core Team, 2014).

Next we used the gpagen function in version 2.1 of the geomorph package (Adams et al., 2014; Adams and Otárola-Castillo, 2013) to run a general Procrustes alignment (Rohlf and Marcus, 1993) of the landmark coordinates while sliding the semilandmarks by minimising Procrustes distance (Bookstein, 1997). We used these Procrustes-aligned coordinates of all specimens to calculate average shape values for each species which we then used for a principal components (PC) analysis with the plotTangentSpace function (Adams and Otárola-Castillo, 2013). We selected the number of principal component (PC) axes that accounted for $95 \%$ of the variation in the data (Figure 1) and used these axes to estimate morphological diversity in each family.

The majority of tenrec species ( 19 out of 31 in our data) belong to the Microgale (shrew-like) genus that has relatively low morphological diversity (Soarimalala and Goodman, 2011; Jenkins, 2003). This may mask signals of higher morphological diversity among other tenrecs. To test this, we created a subset of the tenrec data that included just five of the Microgale species, each representing one of the five sub-divisions of Microgale outlined by Soarimalala \& Goodman (2011), i.e. small, small-medium, medium, large and long-tailed species. We repeated the general Procrustes alignment described above using this reduced data 
set. We then compared the morphological diversity of the full data set (31 species of tenrec) or a reduced data set with just 17 species of tenrec (five Microgale and 12 non-Microgale species; Figure 1) to that of the 12 species of golden moles.

\section{Estimating morphological diversity}

We grouped the PC scores for tenrecs and golden moles separately so that we could estimate the diversity of each family and then compare the two groups (Figure 1). We compared morphological diversity in two ways. First, we used non parametric multivariate analysis of variance (npMANOVA; Anderson, 2001) to test whether tenrecs and golden moles occupied significantly different positions within the morphospaces defined by the PC axes that accounted for $95 \%$ of the overall variation in the data (e.g. Serb et al., 2011; Ruta et al., 2013). A significant difference between the two families would indicate that they have unique morphologies which do not overlap. Second, we compared morphological diversity within tenrecs to the diversity within golden moles.

Morphological diversity (variation in form) is more commonly referred to as morphological disparity (Foote, 1997). There are many different methods for measuring disparity. Calculations based on summary (principal component) axes of shape variation are popular (e.g. Ruta et al., 2013; Foth et al., 2012; Brusatte et al., 2008; Wainwright, 2007) while other methods include calculating disparity directly from 
Procrustes shape variables (Zelditch et al., 2012) or rate-based approaches which depend on phylogenetic branching patterns (e.g. Price et al., 2013, 2010; $\mathrm{O}^{\prime}$ Meara et al. 2006). There is no single best method of measuring disparity (Ciampaglio et al., 2001) and each method makes different assumptions which are appropriate for different situations. Therefore, for clarity, we have chosen to measure variation in physical form using a clear, easily-interpretable method which captures variation in morphological diversity.

We define morphological diversity as the mean Euclidean distance (sum of squared differences) between each species and its family centroid (Figure 3). This is summarised in the equation below where $n$ is the number of species in the family, $i$ is the number of PC axes and $c$ is the average PC score for each axis (the centroid).

$$
\text { Diversity }=\frac{\sqrt{\Sigma\left(P C n_{i}-P C c_{i}\right)^{2}}}{n}
$$

If tenrecs are more morphologically diverse than golden moles, then they should be more dispersed within the morphospaces and have, on average, higher values of mean Euclidean distance.

One possible issue with these analyses is that the two families have unequal sample sizes: 31 (or a subset of 17 ) tenrec species compared to just 12 golden mole species. Morphological diversity is usually decoupled from taxonomic diversity (e.g. Ruta et al., 2013; Hopkins, 2013) so larger groups are not necessarily more morphologically diverse. However, comparing morphological diversity in tenrecs to the diversity 


\section{PeerJ Reviewing Manuscript}

of a smaller family could still bias the results. We used pairwise permutation tests to account for this potential issue.

We tested the null hypothesis that tenrecs and golden moles have the same morphological diversity (the same mean Euclidean distance to the family centroid). If this is true, when we randomly assign the group identity of each species (i.e. shuffle the "tenrec" and "golden mole" labels) and then re-compare the morphological diversity of the two groups, there should be no significant difference between these results and those obtained when the species are assigned to the correct groupings.

We performed this shuffling procedure (random assignation of group identity) 1000 times and calculated the difference in morphological diversity between the two groups for each permutation. This generated a distribution of 1000 values which are calculations of the differences in morphological diversity under the assumption that the null hypothesis (equal morphological diversity in the two families) is true. This method automatically accounts for differences in sample size because shuffling of the group labels preserves the sample size of each group: there will always be 12 species labelled as "golden mole" and then, depending on the analysis, either 31 or 17 species labelled as "tenrec". Therefore, the Iooo permuted values of differences in morphological diversity create a distribution of the expected difference in diversity between a group of sample size 31 (or 17 in the case of the tenrec data subset) compared to a group of sample size 12 under the null hypothesis that the two groups 
have the same morphological diversity. We compared the observed measures of the differences in morphological diversity between the two families to these null distributions to determine whether there were significant differences after taking sample size into account (two-tailed $t$ test). Data and code for all of our analyses are available on GitHub (Finlay and Cooper, 2015).

\section{Results}

Figure 4 depicts the morphospaces defined by the first two principal component (PC) axes from our principal components analyses (PCAs) of skull and mandible morphologies. The PCAs are based on the average Procrustes-superimposed shape coordinates for skulls in three views (dorsal, ventral and lateral).

To compare morphological diversity in the two families, we used the PC axes which accounted for $95 \%$ of the cumulative variation in each of the skull analyses: dorsal ( $\mathrm{n}=6$ axes), ventral ( $\mathrm{n}=7$ axes) and lateral $(\mathrm{n}=7$ axes). First, we compared the position of each family within the morphospace plots. Tenrecs and golden moles occupy significantly different positions in the dorsal (npMANOVA: $\mathrm{F}_{1,42}=68.13, \mathrm{R}^{2}=0.62$, $\mathrm{p}=0.001$ ), ventral (npMANOVA: $\mathrm{F}_{1,42}=103.33, \mathrm{R}^{2}=0.72, \mathrm{p}=0.001$ ) and lateral (npMANOVA: $\mathrm{F}_{1,42}=76.7, \mathrm{R}^{2}=0.65, \mathrm{p}=0.001$ ) skull morphospaces, indicating that the families have very different, non-overlapping cranial and mandible morphologies (Figure 4). For each analysis, PC1 


\section{PeerJ Reviewing Manuscript}

summarises a morphological change from the foreshortened, "squat" shape of golden mole skulls at one extreme to the rostrally elongated shape of tenrecs (particularly the Microgale) at the other extreme.

Second, we compared the morphological diversity within each family. Based on our measures of mean Euclidean distance to the family centroids, tenrec skulls are more morphologically diverse than golden mole skulls when they are measured in lateral view but not in dorsal or ventral view (Table 1). In contrast, when we analysed morphological diversity of skulls within the sub-sample of 17 tenrecs (including just five Microgale species) compared to the 12 golden mole species, we found that tenrec skulls were significantly more morphologically diverse than golden moles in all analyses (Table 1). The pairwise permutation tests for each analysis confirmed that differences in morphological diversity were not artefacts of differences in sample size (Table 2)

\section{Discussion}

Tenrecs are often cited as an example of a mammalian group with high morphological diversity (Olson, 2013; Soarimalala and Goodman, 2011; Eisenberg and Gould, 1969). They are also more ecologically diverse than their closest relatives (Soarimalala and Goodman, 2011; Bronner, 1995) so we predicted that they would be more morphologically diverse than golden moles. However, our results do not support our original prediction, highlighting the importance of quantitative tests of perceived 


\section{PeerJ Reviewing Manuscript}

morphological patterns.

In our full analysis, tenrecs only had higher morphological diversity than golden moles when the skulls were measured in lateral view (Table 1). There was no difference in morphological diversity when we analysed the skulls in dorsal or ventral views. This is most likely due to our choice of landmarks. The two outline curves in lateral view (Figure 2) emphasise morphological variation in the back and top of the skulls. These curves summarise overall shape variation but they do not identify clear anatomical differences because they are defined by relative features rather than homologous structures (Zelditch et al., 2012). Therefore, high morphological diversity in tenrecs when analysed in this view may not indicate biologically or ecologically relevant variation. These lateral aspects of the skull morphology were not visible in the dorsal and ventral photographs so they could not be included in those analyses. In contrast, our landmarks in the dorsal, and particularly ventral, views focus on morphological variation in the overall outline shape of the sides of the skull and palate (Figure 2). The result that tenrecs are no more diverse than golden moles in these areas makes intuitive sense: most tenrecs have non-specialised insectivorous or faunivorous diets (Olson, 2013) so there is no obvious functional reason why they should have particularly diverse palate morphologies. Similarly, while there are anatomical differences among tenrec tooth morphologies (Asher and Sánchez-Villagra, 2005) more work is required to determine if and how those differences correspond to variation in diet or feeding ecology. The different results for our analysis of lateral skull morphologies compared 


\section{PeerJ Reviewing Manuscript}

to dorsal and ventral views highlight the importance of using multiple approaches when studying $3 \mathrm{D}$ morphological shape using $2 \mathrm{D}$ geometric morphometrics techniques (Arnqvist and Mårtensson, 1998). Future analyses could use $3 \mathrm{D}$ geometric morphometric approaches to test whether similar patterns emerge.

Landmark choice and placement will inevitably influence the results of a geometric morphometrics study. Our interest in broad-scale, cross-taxonomic comparisons of cranial morphology constrained our choice of landmarks to those that could be accurately identified in many different species (e.g. Ruta et al., 2013; Goswami et al., 2011; Wroe and Milne, 2007; Goswami, 2006). In contrast, studies that use skulls to characterise morphological variation within species (e.g. Blagojević and Milošević-Zlatanović, 2011; Giannini et al., 2010; Flores et al., 2010; Bornholdt et al., 2008) or to delineate species boundaries within a clade (e.g. Panchetti et al., 2008) tend to focus on more detailed, biologically homologous landmarks (Zelditch et al., 2012). Repeating our analyses with a narrower taxonomic focus may give greater insight into the specific morphological differences among subgroups of tenrecs and golden moles.

In addition to the differences among the three skull views, our results indicate that, in skulls at least, the overall morphological diversity within tenrecs is not as large as is often assumed (e.g. Eisenberg and Gould, 1969; Olson, 2013). Studies of morphological variation are sensitive to the sampling used. If a particular morphotype 


\section{PeerJ Reviewing Manuscript}

is over-represented then the similarities among those species will reduce the overall morphological variation within the group (Foote, 1991). This appears to be the case for our data; it was only when we included a sub-sample of Microgale tenrecs that we found higher morphological diversity in tenrecs compared to golden moles across all three skull analyses (Table 1). While there are clear physical differences among family members (Olson, 2013; Eisenberg and Gould, 1969), the majority of tenrecs (the Microgale) are very morphologically similar (Jenkins, 2003) so morphological diversity in the family as a whole is not as large as it first appears.

The goal of our study was to quantify morphological variation in tenrecs instead of relying on subjective assessments of their high morphological diversity. However, it is difficult to quantify overall morphological diversity because any study is inevitably constrained by its choice of specific traits (Roy and Foote, 1997). While the skull is widely regarded as a good model for studying morphological variation (e.g. Blagojević and Milošević-Zlatanović, 2011; Flores et al., 2010; Giannini et al., 2010), quantifying variation in other morphological traits could yield different patterns. Therefore future work should extend our approach beyond skulls to gain a more complete understanding of the overall morphological diversity of tenrecs and golden moles. While recognising these limitations, our results provide valuable insights into the differences between subjective and quantitative assessments of morphological diversity. 


\section{PeerJ Reviewing Manuscript}

\section{Conclusions}

We have presented the first quantitative investigation of morphological diversity in tenrecs. Our results indicate that, overall, tenrec skulls are not more morphologically diverse than golden moles and that similarities among the species rich Microgale tenrecs mask signals of higher morphological diversity among the rest of the family. Of course the results presented here are restricted to just one aspect of morphological variation and further analysis of other traits is required. However, our findings provide a foundation for future investigations and represent a significant step towards a more quantitative understanding of patterns of morphological and evolutionary diversity in tenrecs.

\section{Acknowledgements}

We thank Thomas Guillerme, François Gould and the members of NERD club for their insightful discussions and comments. We are grateful to the Editor, Nick Crumpton and an anonymous reviewer for their constructive comments. Thank you to museum staff and curators for facilitating our access to their collections: Leona Leonard and Nigel Monaghan (Natural History Museum, Ireland), Roberto Portela Miguez and Paula Jenkins (Natural History Museum, London), Esther Langan (Smithsonian Institute NMNH), Eileen Westwig (American Museum of Natural History), Judy Chupasko (Museum of Comparative Zoology) and Bill Stanley and Steve Goodman (Field Museum). 


\section{PeerJ Reviewing Manuscript}

\section{References}

Adams, D. C., C. M.L., E. Otárola-Castillo, and E. Sherratt. 2014. geomorph: Software for geometric morphometrics analyses. $\mathrm{r}$ package version 2.1.

http://cran.r-project.org/web/packages/geomorph/index.html.

Adams, D. C. and E. Otárola-Castillo. 2013. geomorph: an R package for the collection and analysis of geometric morphometric shape data. Methods in Ecology and Evolution 4:393-399.

Adams, D. C., F. J. Rohlf, and D. Slice. 2004. Geometric morphometrics: Ten years of progress following the "revolution". Italian Journal of Zoology 71:5-16.

Anderson, M. 2001. A new method for non-parametric multivariate analysis of variance. Austral Ecology 26:32-46.

Arnqvist, G. and T. Mårtensson. 1998. Measurement error in geometric morphometrics; empirical strategies to assess and reduce its impact on measures of shape. Acta Zoologica Academiae Scientiarum Hungaricae 44:73-96.

Asher, R. J. and T. Lehmann. 2008. Dental eruption in Afrotherian mammals. BMC Biology 6:14.

Asher, R. J. and M. R. Sánchez-Villagra. 2005. Locking yourself out: diversity among dentally zalambdodont therian mammals. Journal of Mammalian Evolution 12:265-282. 


\section{PeerJ Reviewing Manuscript}

Barrow, E. and N. Macleod. 2008. Shape variation in the mole dentary (Talpidae: Mammalia). Zoological Journal of the Linnean Society 153:187-211.

Blagojević, M. and S. Milošević-Zlatanović. 2011. Sexual shape dimorphism in Serbian roe deer (Capreolus capreolus L.). Mammalian Biology - Zeitschrift für Säugetierkunde 76:735-740.

Bookstein, F. 1997. Landmark methods for forms without landmarks: morphometrics of group differences in outline shape. Medical Image Analysis 1:225-243.

Bornholdt, R., L. R. Oliveira, and M. E. Fabián. 2008. Size and shape variability in the skull of Myotis nigricans (schinz, 1821) (chiroptera: Vespertilionidae) from two geographic areas in brazil. Brazilian Journal of Biology 68:623-631.

Bronner, G. 1995. Systematic revision of the golden mole genera Amblysomus, Chlorotalpa and Calcochloris (Insectivora:

Chrysochloromorpha; Chrysochloridae). Ph.D. thesis.

Brusatte, S., M. Benton, M. Ruta, and G. Lloyd. 2008. Superiority, competition and opportunism in the evolutionary radiation of dinosaurs. Science 321:1485-1488.

Canon. 2013. Digital photo professional version 3.11.30.3.

Cardini, A. 2014. Missing the third dimension in geometric 
morphometrics: how to assess if $2 \mathrm{~d}$ images really are a good proxy for 3d structures? Hystrix 25:73-81.

Ciampaglio, C., M. Kemp, and D. McShea. 2001. Detecting changes in morphospace occupation patterns in the fossil record: characterization and analysis of measures of disparity. Paleobiology 27:695-715.

Davis, W. and D. Schmidly. 1997. The Mammals of Texas - Online Edition. http://www.nsrl.ttu.edu/tmot1/Default.htm.

Drake, A. and C. Klingenberg. 2010. Large-scale diversification of skull shape in domestic dogs: disparity and modularity. The American naturalist 175:289-301 10.1086/650372.

Eisenberg, J. F. and E. Gould. 1969. The Tenrecs: A Study in Mammalian Behaviour and Evolution. Smithsonian Contributions to Zoology 27:1-152.

Finlay, S. and N. Cooper. 2013a. "Insectivore" mammal skulls, dorsal view. http://dx.doi.org/10.6084/m9.figshare.705863.

Finlay, S. and N. Cooper. 2013b. "Insectivore" mammal skulls, lateral view. http://dx.doi.org/10.6084/m9.figshare.715890.

Finlay, S. and N. Cooper. 2013c. "Insectivore" mammal skulls, ventral view. http://dx.doi.org/10.6084/m9.figshare.715841.

Finlay, S. and N. Cooper. 2015. GitHub data and code repository. https://github.com/SiveFinlay/Diversity_Paper. 


\section{PeerJ Reviewing Manuscript}

Flores, D., F. Abdala, and N. Giannini. 2010. Cranial ontogeny of Caluromys philander (Didelphidae: Caluromyinae): a qualitative and quantitative approach. Journal of Mammalogy 91:539-550 10.1644/o9-MAMM-A-291.1.

Foote, M. 1991. Morphological and taxonomic diversity in a clade's history: the blastoid record and stochastic simulations. University of Michigan, Museum of Paleontology Contributions 28:101-140.

Foote, M. 1997. The evolution of morphological diversity. Annual Review of Ecology and Systematics 28:129-152.

Foth, C., S. Brusatte, and R. Butler. 2012. Do different disparity proxies converge on a common signal? Insights from the cranial morphometrics and evolutionary history of Pterosauria (Diapsida: Archosauria). Journal of Evolutionary Biology 25:904-915.

Garbutt, N. 1999. Mammals of Madagascar. Pica Press, Sussex.

Giannini, N., V. Segura, M. Giannini, and D. Flores. 2010. A quantitative approach to the cranial ontogeny of the puma. Mammalian Biology Zeitschrift für Säugetierkunde 75:547 - 554.

Goodman, S. M., C. J. Raxworthy, C. P. Maminirina, and L. E. Olson. 2006. A new species of shrew tenrec (Microgale jobihely) from northern Madagascar. Journal of Zoology 270:384-398.

Goswami, A. 2006. Cranial modularity shifts during mammalian evolution. The American Naturalist 168:270-280. 


\section{PeerJ Reviewing Manuscript}

Goswami, A., N. Milne, and S. Wroe. 2011. Biting through constraints: cranial morphology, disparity and convergence across living and fossil carnivorous mammals. Proceedings of the Royal Society B: Biological Sciences 278:1831-1839.

Hoffmann, R. and D. Lunde. 2008. Order Erinaceomorpha Pages 192-297. Princeton University Press, Oxfordshire, UK.

Hopkins, M. 2013. Decoupling of taxonomic diversity and morphological disparity during decline of the Cambrian trilobite family Pterocephaliidae. Journal of Evolutionary Biology 26:1665-1676.

Jenkins, P. 2003. Microgale, shrew tenrecs Pages 1273-1278. The University of Chicago Press, Chicago.

Karataş, A., M. Mouradi Gharkheloo, and T. Kankiliç. 2007. Cranial features and karyotypes of two hedgehogs (Insectivora: Erinaceidae) from Iran. Anatomia, Histologia, Embryologia 36:419-423.

Knox Jones, J. and R. Manning. 1992. Insectivores Page 75. Texas Tech University Press, United States of America.

Lin, L.-K. and M. Motokawa. 2010. Mammals of Taiwan, Volume 1. Soricomorpha. http://mammal . biota.biodiv.tw/

Losos, J. B. and D. Mahler. 2010. Adaptive radiation: the interaction of ecological opportunity, adaptation and speciation Pages 381-420. Sinauer Association, Sunderland, MA. 


\section{PeerJ Reviewing Manuscript}

MacLeod, N. 2012. Going Round the Bend ii: Extended Eigenshape Analysis. http://www . palass .org

MacPhee, R. 1987. The shrew tenrecs of Madagascar: Systematic revision and holocene distribution of Microgale (Tenrecidae, Insectivora). American Museum Novitates Number 2889:1-45.

McGee, M. D. and P. C. Wainwright. 2013. Convergent evolution as a generator of phenotypic diversity in threespine stickleback. Evolution 67:1204-1208.

Mitteroecker, P. and P. Gunz. 2009. Advances in geometric morphometrics. Evolutionary Biology 36:235-247.

Muldoon, K., D. de Blieux, E. Simons, and P. Chatracth. 2009. The subfossil occurrence and paleoecological significance of small mammals at Ankilitelo Cave, Southwestern Madagascar. Journal of Mammalogy 90:111-1131.

Muschick, M., A. Indermaur, and W. Salzburger. 2012. Convergent evolution within an adaptive radiation of cichlid fishes. Current Biology 22:1-7.

Nagorsen, D. 2002. An identification manual to the small mammals of British Columbia. Ministry of Sustainable Resource Management, Ministry of Water, Land and Air Protection, Biodiversity Branch and Royal BC Museum. 


\section{PeerJ Reviewing Manuscript}

Nowak, R. 1983. Walker's Mammals of the World, 4th edition vol. 1. Johns Hopkins University Press, Baltimore.

Olson, L. E. 2013. Tenrecs. Current Biology 23:R5-R8.

Olson, L. E. and S. M. Goodman. 2003. Phylogeny and biogeography of tenrecs Pages 1235-1242. The University of Chicago Press, Chicago.

O'Meara, B., C. Ané, M. Sanderson, and P. Wainwright. 2006. Testing for different rates of continuous trait evolution using likelihood. Evolution 60:922-933.

Panchetti, F., M. Scalici, G. Carpaneto, and G. Gibertini. 2008. Shape and size variations in the cranium of elephant-shrews: a morphometric contribution to a phylogenetic debate. Zoomorphology 127:69-82.

Poux, C., O. Madsen, J. Glos, W. W. de Jong, and M. Vences. 2008.

Molecular phylogeny and divergence times of Malagasy tenrecs: Influence of data partitioning and taxon sampling on dating analyses. BMC Evolutionary Biology 8:102.

Price, S., J. Tavera, T. Near, and P. Wainwright. 2013. Elevated rates of morphological and functional diversification in reef-dwelling haemulid fishes. Evolution 67:417-428.

Price, S., P. Wainwright, D. Bellwood, E. Kazancioglu, D. Collar, and T. Near. 2010. Functional innovations and morphological diversification in parrotfish. Evolution 64:3057-3068. 


\section{PeerJ Reviewing Manuscript}

Quérouil, S., P. Hutterer, M. Colyn, J. Kerbis Peterhans, and E. Verheyen. 2001. Phylogeny and evolution of African shrews (Mammalia: Soricidae) inferred from 16s rRNA sequences. Molecular Phylogenetics and Evolution 20:185-195.

R Core Team. 2014. R: A language and environment for statistical computing. http://www.R-project.org/.

Repenning, C. 1967. Subfamilies and Genera of the Soricidae. Geological Survey Professional Paper 565 United States Government Printing Office, Washington.

RHOI. 2013. Revealing Hominid Origins Iinitiative Fossil Photography Protocol, U.C Berkeley.http://rhoi.berkeley.edu/RHOI_photo/ RHOI_Photography_Protocol.html.

Rohlf, F. 2012. Tpsutil ver 1.53. http://life.bio.sunysb.edu/morph/.

Rohlf, F. 2013. Tpsdig2 ver 2.17. http://life.bio.sunysb.edu/morph/,

Rohlf, J. and L. Marcus. 1993. A revolution in morphometrics. Trends in Ecology and Evolution 8:129-132.

Roy, K. and M. Foote. 1997. Morphological approaches to measuring biodiversity. Trends in Ecology and Evolution 12:277-281.

Ruta, M., K. Angielczyk, J. Fröbisch, and M. Benton. 2013. Decoupling of morphological disparity and taxic diversity during the adaptive radiation of anomodont therapsids. Proceedings of the Royal Society B: Biological Sciences 280:20131071. 


\section{PeerJ Reviewing Manuscript}

Salton, J. A. and E. Sargis. 2009. Evolutionary morphology of the Tenrecoidea (Mammalia) hindlimb skeleton. Journal of Morphology 270:367-387.

Serb, J., A. Alejandrino, E. Otárola-Castillo, and D. Adams. 2011. Morphological convergence of shell shape in distantly related scallop species (Mollusca: Pectinidae). Zoological Journal of the Linnean Society 163:571-584.

Soarimalala, V. and S. Goodman. 2011. Les petits mammiferes de Madagascar. Guides sur la diversité biologique de Madagascar Association Vahatra, Antananarivo, Madagascar.

Wainwright, P. 2007. Functional versus morphological diversity in macroevolution. Annual Review of Ecology, Evolution, and Systematics 38:381-401.

Wilson, D. and D. Reeder. 2005. Mammal species of the world. A taxonomic and geographic reference (3rd edition). Johns Hopkins University Press.

Wroe, S. and N. Milne. 2007. Convergence and remarkably consistent constraint in the evolution of carnivore skull shape. Evolution 61:1251-1260.

Zelditch, M., D. Swiderski, and D. Sheets. 2012. Geometric Morphometrics for Biologists, 2nd edition. Academic Press, Elsevier. 


\section{1}

Flowchart diagram of data collection and analysis

Summary of the main steps in our data collection, processing and analysis protocol. Note that the analyses were repeated separately for each set of photographs: skulls in dorsal, ventral and lateral views. The dashed arrows refer to the stage at which we selected a subsample of the tenrecs (including just five species of the Microgale genus) so that we could compare the morphological diversity of this reduced subsample of tenrec species to the diversity of golden moles. 


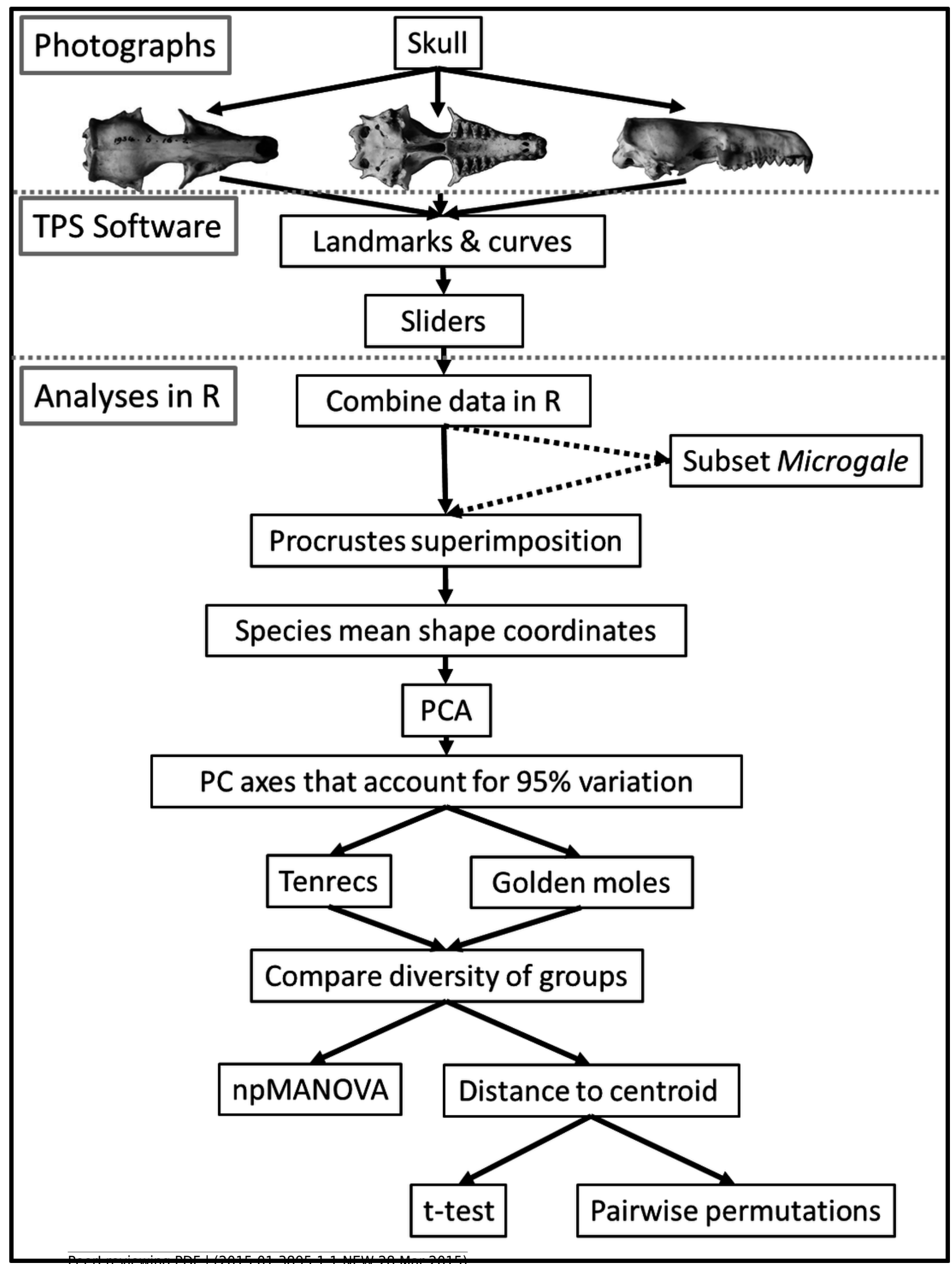


2

Skulls: dorsal, ventral and lateral landmarks

Landmarks (numbered points) and curves (outlines) for the skulls in dorsal (A),ventral (B) and lateral (C) view. See the supplementary material for detailed landmark descriptions. The skulls are an example of a Potamogale velox (otter shrew tenrec), museum accession number BMNH 1934.6.16.2. 
PeerJ Reviewing Manuscript
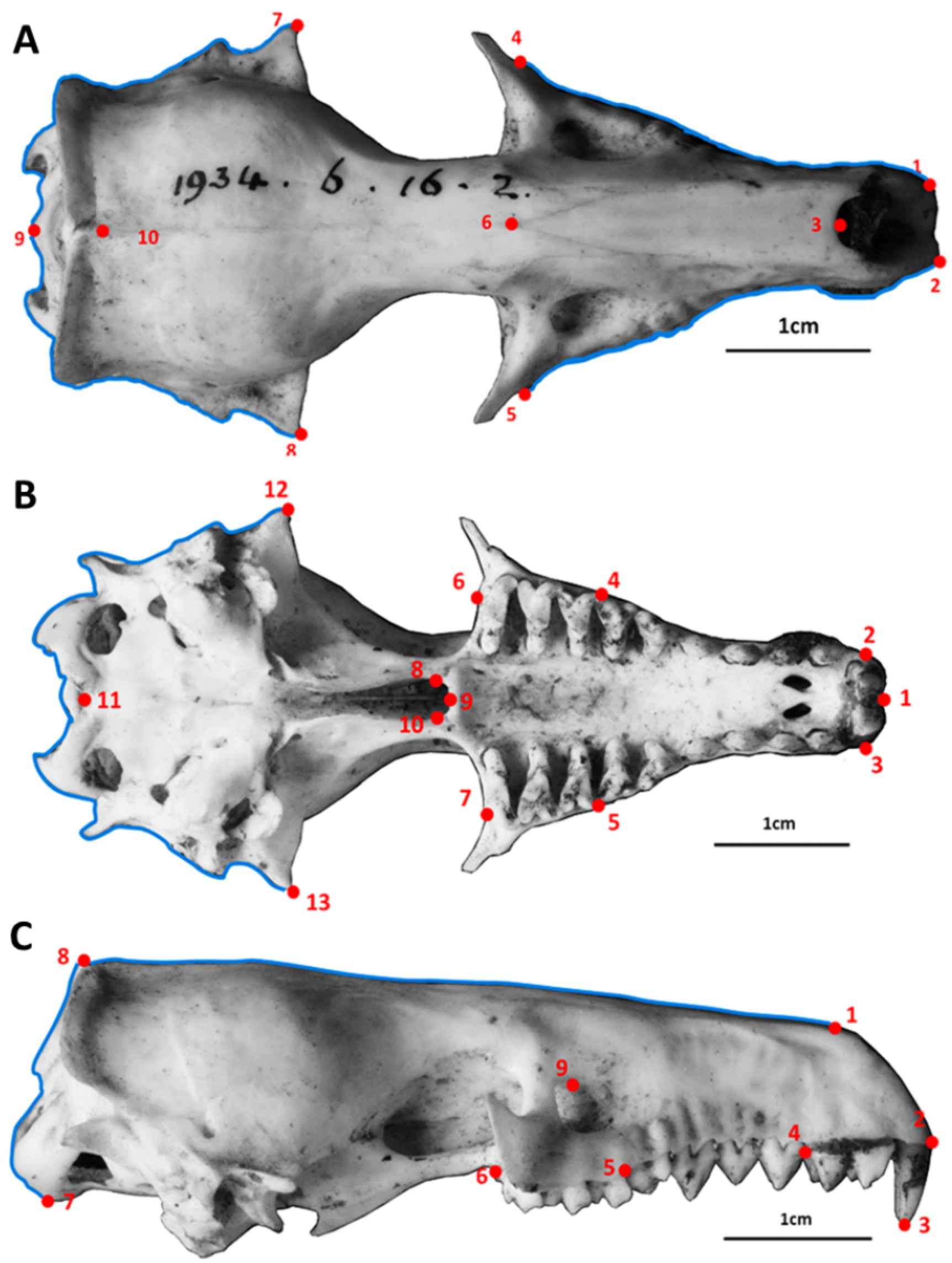


\section{3}

Calculating diversity as mean Euclidean distance to Family centroid.

Estimating morphological diversity as the mean Euclidean distance between each species and the Family centroid. Every species had scores on the principal components (PC) axes that accounted for $95 \%$ of the variation in the principal components analysis. The number of axes $(\mathrm{PCn})$ varied for each analysis but they were the same within a single analysis. PC scores were used to calculate the Euclidean distance from each species to the Family centroid (average PC scores for the entire Family). Morphological diversity of the Family is the average value of these Euclidean distances. 


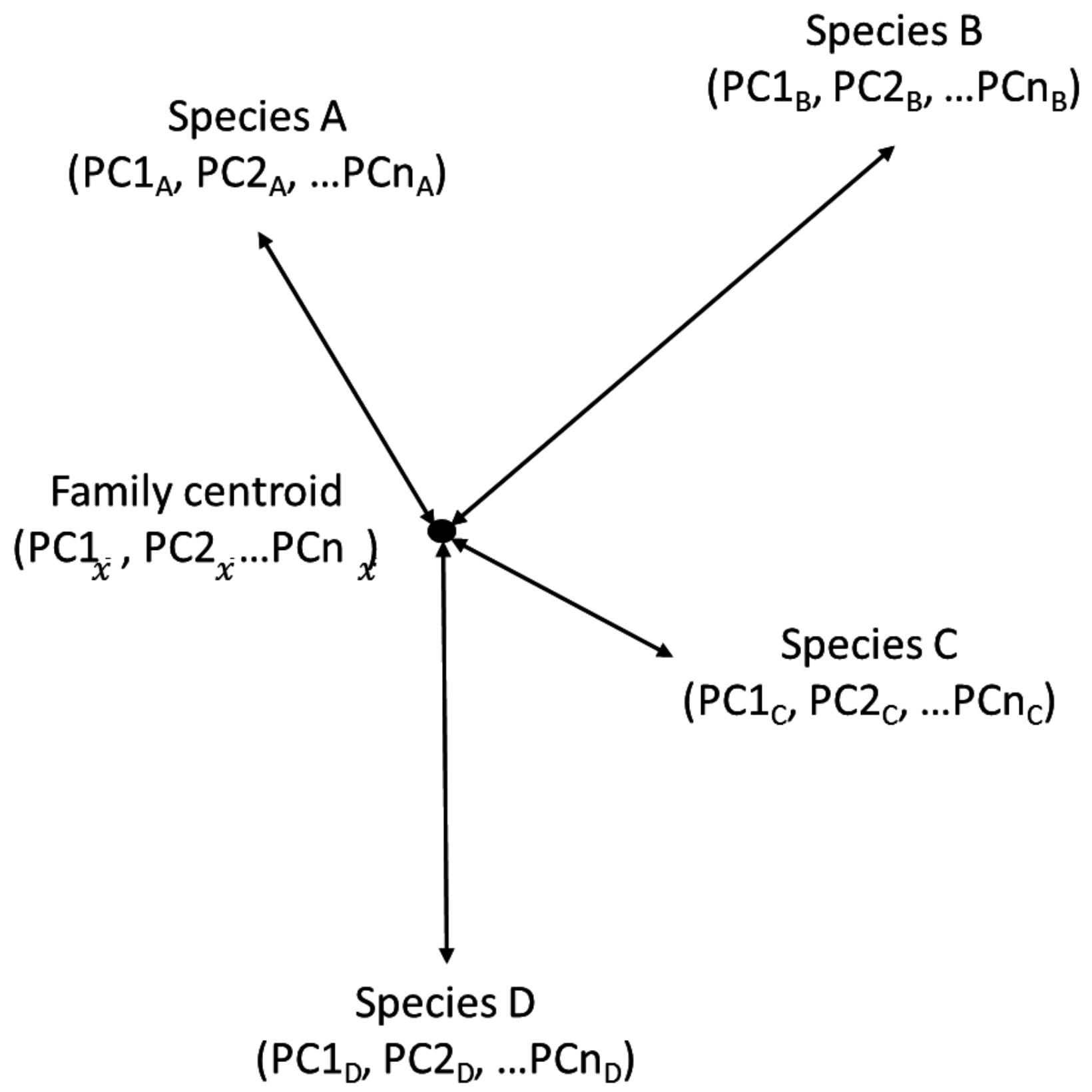


4

Morphospace (principal components) plot of morphological diversity in tenrec and golden mole skulls

Principal components plots of the morphospaces occupied by tenrecs (triangles, $n=31$ species) and golden moles (circles, $n=12$ species) for skulls in dorsal (A), ventral (B) and lateral (C) views. Each point represents the average skull shape of an individual species. Axes are principal component 1 (PC1) and principal component 2 (PC2) of the average scores from principal components analyses of mean Procrustes shape coordinates for each species.

A

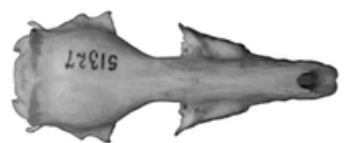

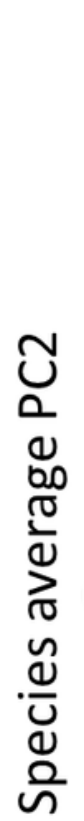
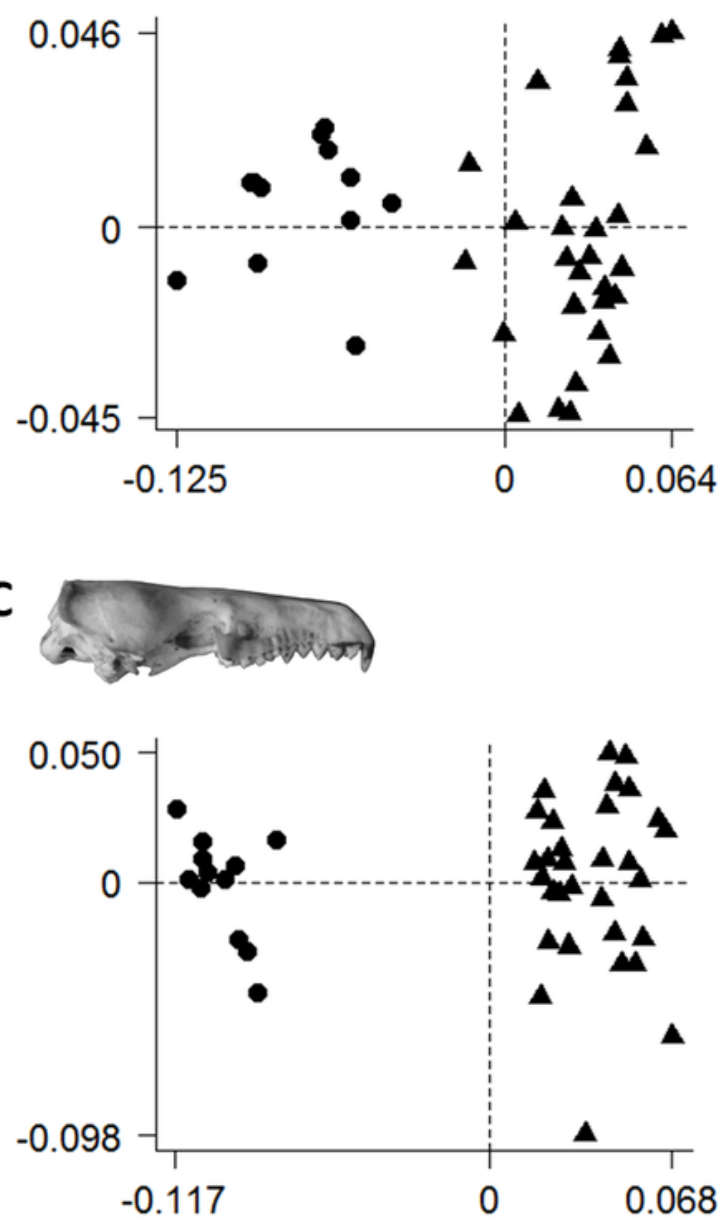

B
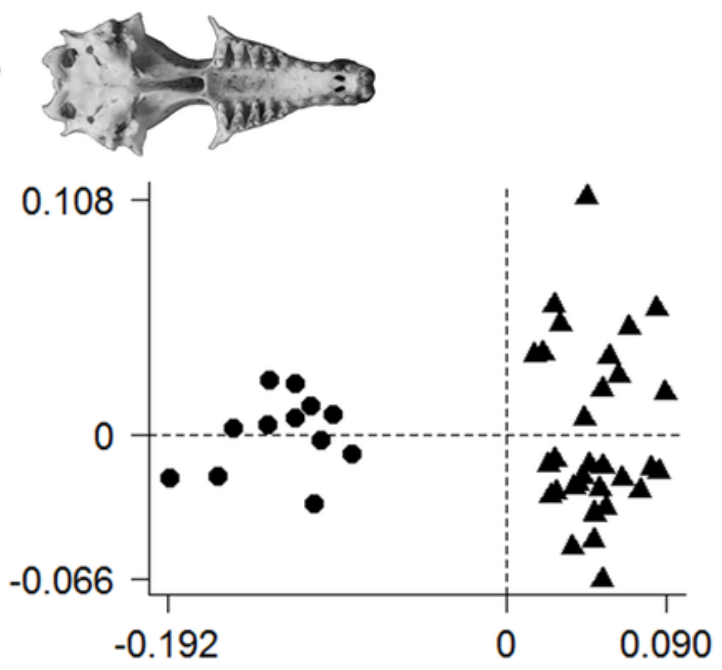

Tenrecs

Golden Moles 


\section{Table $\mathbf{1}_{\text {(on next page) }}$}

Comparing morphological diversity in tenrecs and golden moles

Morphological diversity in tenrecs compared to golden moles (12 species). $\mathrm{N}$ is the number of tenrec species: 31 species or 17 species including just five representatives of the Microgale genus. Morphological diversity of the family is the mean Euclidean distance from each species to the family centroid. Significant differences between the two Families $(p<0.05)$ from two-tailed t-tests are highlighted in bold. 


\begin{tabular}{|c|c|c|c|c|c|}
\hline \multirow[t]{3}{*}{$\mathrm{N}$} & \multirow[t]{3}{*}{ Analysis } & \multicolumn{2}{|c|}{ Morphological diversity } & \multirow[t]{3}{*}{$t_{d f}$} & \multirow[t]{3}{*}{$\mathrm{p}$ value } \\
\hline & & Tenrecs & Golden moles & & \\
\hline & & $($ mean \pm s.e $)$ & $($ mean \pm s.e $)$ & & \\
\hline \multirow[t]{3}{*}{31} & Skulls dorsal & $0.036 \pm 0.0029$ & $0.029 \pm 0.0032$ & -1.6329 .88 & 0.11 \\
\hline & Skulls ventral & $0.048 \pm 0.0034$ & $0.044 \pm 0.0041$ & $-0.68_{26.99}$ & 0.51 \\
\hline & Skulls lateral & $0.044 \pm 0.0041$ & $0.032 \pm 0.0037$ & $-2.16_{35.03}$ & 0.04 \\
\hline \multirow[t]{3}{*}{17} & Skulls dorsal & $0.044 \pm 0.0025$ & $0.029 \pm 0.0032$ & $-3.62_{22.75}$ & $<\mathbf{0 . 0 1}$ \\
\hline & Skulls ventral & $0.054 \pm 0.0039$ & $0.042 \pm 0.0041$ & -2.2325 .46 & 0.04 \\
\hline & Skulls lateral & $0.054 \pm 0.0053$ & $0.031 \pm 0.0037$ & $-3 \cdot 4726.31$ & $<\mathbf{0 . 0 1}$ \\
\hline
\end{tabular}




\section{Table 2 (on next page)}

Results of the permutation tests

Results of the permutation analyses comparing the observed differences in morphological diversity to a null distribution of expected results. Morphological diversity of the family is the mean Euclidean distance from each species to the family centroid. Results are shown for both the full ( $\mathrm{N}=31$ species of tenrec compared to 12 species of golden mole) and reduced $(\mathrm{N}=17$ species of tenrec compared to 12 golden moles) data sets. Significant values $(\mathrm{p}<0.05)$ indicate that the observed morphological diversity is different to the expected differences under a null hypothesis of equivalent diversities in the two Families. 


\begin{tabular}{cccccccc}
\hline N & Analysis & \multicolumn{4}{c}{ Morphological diversity } & \multirow{2}{*}{ p value } \\
\hline & & \multicolumn{3}{c}{ Measured values } & \multicolumn{2}{c}{ Permuted values } & \\
\cline { 3 - 7 } & & Tenrecs & Golden moles & Difference & Min. & Max. & \\
\hline \multirow{2}{*}{31} & Dorsal & 0.036 & 0.029 & 0.007 & -0.011 & 0.009 & $\mathbf{0 . 0 1 3}$ \\
& Ventral & 0.048 & 0.044 & 0.004 & -0.014 & 0.013 & $\mathbf{0 . 0 2 3}$ \\
& Lateral & 0.044 & 0.032 & 0.012 & -0.012 & 0.011 & $<\mathbf{0 . 0 0 1}$ \\
\hline \multirow{2}{*}{17} & Dorsal & 0.044 & 0.029 & 0.015 & -0.011 & 0.014 & $<\mathbf{0 . 0 0 1}$ \\
& Ventral & 0.054 & 0.042 & 0.013 & -0.017 & 0.019 & $\mathbf{0 . 0 2 3}$ \\
& Lateral & 0.054 & 0.031 & 0.022 & -0.018 & 0.019 & $<\mathbf{0 . 0 0 1}$ \\
\hline
\end{tabular}

